\title{
Characterization of Coronary Plaque in Psoriasis and the Impact of Current Therapies
}

\author{
Ashlee L Culver ${ }^{1}$, Annika S Silfvast-Kaiser ${ }^{2}$, Alan Menter ${ }^{1,2 *}$ \\ 'Texas A\&M Health Science Center College of Medicine, TX \\ ${ }^{2}$ Division of Dermatology, Baylor University Medical Center, Dallas, TX
}

Article Info

\section{Article Notes}

Received: July 25, 2018

Accepted: September 18, 2018

\section{*Correspondence:}

Dr. Alan Menter, MD, FAAD, 3900 Junius St, Suite 145 Dallas, TX 75246; Telephone No: 972-386-7546;

E-mail: amderm@gmail.com.

(c) 2018 Menter A. This article is distributed under the terms of the Creative Commons Attribution 4.0 International License.

\section{Key words:}

Psoriasis

Myocardial infarction

Coronary artery plaque

Cardiovascular disease

Coronary artery disease

Atherosclerosis

\begin{abstract}
Psoriasis is a systemic inflammatory disease which contributes to an increased risk for cardiovascular disease, specifically coronary artery disease. Patients with psoriasis tend to have greater total coronary plaque burden and more high risk plaque than healthy controls. This likely contributes to the higher rate of myocardial infarction and 4-5 year reduction in lifespan observed in our psoriasis population. With biologic therapy and improvement in PASI scores, total plaque burden and noncalcified coronary plaque decreases as well. Specifically, ustekinumab decreases intima-media thickness and reduces vascular inflammation. Likewise, TNF- $\alpha$ inhibitors decrease vascular inflammation and reduce cardiovascular events in both sexes, and reduce coronary plaque formation in men with psoriasis. This may be due to elevation in glycoprotein acetylation, which is associated with cardiovascular events and elevated in psoriasis. This elevation has also been shown to decrease with adalimumab usage. Despite all of the knowledge gained on this topic, the incidence of myocardial infarction in psoriasis patients currently remains unchanged when compared to prior years. Consequently, we emphasize the need for further research on the unique pathogenesis of psoriatic coronary plaque formation as well as the effect biologic agents have on this coronary plaque in order to improve the wellbeing of this patient population.
\end{abstract}

\section{Introduction}

Psoriasis is a T-cell mediated inflammatory disorder with estimates of prevalence ranging from $0.51 \%$ to $11.43 \%$ in adults worldwide $^{1}$. The erythematous, scaly plaques characteristic of chronic plaque psoriasis are not only unsightly and uncomfortable for patients, but they also represent the possibility of internal manifestations of the disease. The many comorbidities associated with psoriasis include, butare not limited to, joint disease, depression, metabolic syndrome, and cardiovascular disease ${ }^{2,3}$. The increased risk for cardiovascular disease has been well documented ${ }^{4-6}$ and is thought to be due to chronic systemic inflammation. This conclusion is based on the understanding that T-helper cells play a central role in the pathogenesis of both atherosclerosis and psoriasis ${ }^{7,8}$. A surge of research regarding this topic has occurred as the association between psoriasis and cardiovascular disease becomes more widely appreciated. This has resulted in better characterization of cardiovascular risk in these patients, which unfortunately includes a shorter average lifespan. In psoriasis patients with a body surface area $(\mathrm{BSA})>10 \%$, the risk of death has been shown to be significantly increased by about 1.8 times when compared to matched controls without psoriasis. Although a significant increase in mortality has not been definitively shown for those with less than $10 \%$ BSA, these 
patients may still be at a higher risk for comorbidities such as chronic kidney disease, chronic obstructive pulmonary disease, diabetes, and history of myocardial infarction ${ }^{9}$. In addition, young patients with severe psoriasis actually have the greatest risk for having a myocardial infarction associated with their disease, even after accounting for other traditional risk factors and concomitant health conditions ${ }^{10}$. Therefore, psoriasis has been named an independent risk factor for myocardial infarction ${ }^{10}$.

\section{Coronary Artery Disease Burden in Psoriasis}

Researchers and clinicians have sought to understand more about coronary artery disease and ways to diagnose and characterize coronary artery plaque in psoriasis patients. Interest has peaked regarding this topic after multiple studies have showed an increased risk of acute coronary syndrome in psoriasis patients, specifically, myocardial infarction ${ }^{5,10}$. Using coronary angiography, Armstrong et al. was able to establish that psoriasis patients had a higher prevalence of coronary artery disease than their controls, especially with a disease duration of 8 years or greater ${ }^{11}$. In order to assess subclinical atherosclerosis, Mansouri et al. compared coronary artery calcium scores of type 2 diabetics to psoriasis patients with moderate to severe disease activity. This study showed that when considering mean total Agatston scores, both groups had similar amounts of coronary artery calcium present which were 3 times higher than a normal control group ${ }^{12}$.

\section{Coronary Artery Plaque Characterization}

In order to more completely understand true cardiovascular risk, it is important to not only assess the severity of the coronary plaque in psoriasis patients but to also characterize the plaque itself. This allows clinicians to know how to assess the risk in this population to improve diagnostic and prognostic ability. It has been shown that patients with systemic inflammatory diseases, such as HIV, tend to have more noncalcified plaque and high risk plaque $^{13,14}$. In the same way a small study by Bissonnette et al. showed that patients with psoriasis tend to have an increase in mixed plaques (calcified and noncalcified), as well as a greater mean overall burden of coronary atherosclerosis when compared to controls. ${ }^{15}$ This is true even after adjusting for factors like obesity ${ }^{15}$.

Recently, Circulation published a much larger study, with the goal of using coronary CT angiography to characterize coronary artery plaque in psoriasis when compared to a cohort of hyperlipidemic patients and healthy volunteers. The study demonstrated that psoriasis patients had a significantly greater total coronary plaque burden when compared to the healthy volunteers, as well as greater noncalcified plaque burden than both the hyperlipidemic patients and healthy controls ${ }^{16}$. They had more high risk plaque than healthy individuals as well ${ }^{16}$.
In addition, after one year of biologic therapy, patients who experienced significant improvement in the Psoriasis Area and Severity Index (PASI) score showed a decreased total coronary plaque burden $(\beta=0.45,95 \%$ CI: $0.23-$ $0.67 ; \mathrm{p}<0.001)$ and noncalcified coronary plaque burden $(\beta=0.53,0.32-0.74 ; p<0.001)$. These values were adjusted for confounding variables such as age, sex, atherosclerotic cardiovascular disease 10-year risk, body mass index, lipid lowering therapy, and systemic/biologic psoriasis treatment. However, in patients who had worsening of their psoriasis, there was an increase in noncalcified coronary artery plaque burden concomitantly. The levels of tumor necrosis factor-alpha (TNF- $\alpha$ ) and other inflammatory proteins in patients with improved PASI scores also decreased significantly. Nevertheless, treatment with biologics did not affect insulin resistance or cholesterol efflux capacity in the psoriasis cohort. ${ }^{16}$ Shen et al. used CT angiography to assess coronary atherosclerosis in patients with psoriatic arthritis. Using this method, the researchers discovered that three-vessel disease occurred at a much higher frequency in patients with psoriatic arthritis than in the control group. Longer disease duration was associated with mixed plaque and noncalcified plaque formation in the psoriatic arthritis patients, which are understood to be more vulnerable plaque subtypes ${ }^{17}$.

\section{Plaque Formation on the Molecular Level and Targeted Treatments}

Along with the concern for coronary artery disease in psoriasis, comes an interest in understanding more about the pathogenesis of atherosclerosis in these patients at the molecular level, with the hope of offering more targeted treatment options in the future. T-cells are central to the pathogenesis of psoriasis, and they can become stimulated to differentiate into T-helper 1 cells which produce cytokines such as IL-17, TNF- $\alpha$, and IL$23^{18,19}$. These cytokines are known to play a crucial role in driving psoriasis, which is why many current therapies target them specifically. The cytokine IL-36y has recently been linked to psoriasis and angiogenesis as it induces IL-23 which promotes both processes ${ }^{19}$. Although drugs targeting IL-36y have not been developed yet, anti-IL-23 biologic agents are available, including ustekinumab and guselkumab. Ustekinumab, the anti- IL-12/23 medication, has been shown to decrease intima-media thickness, thus promoting the notion that it may improve cardiovascular disease in psoriasis patients ${ }^{20}$. Ustekinumab treatment has also been associated with decreased systemic and vascular inflammation determined by using standardized uptake values with F-fluorodeoxyglucose PET/CT after achieving a reduction in PASI score of $75 \%{ }^{21}$. Guselkumab, a biologic agent that specifically targets IL-23, has shown extraordinary rates of reduction in skin symptom severity in psoriasis patients since becoming available ${ }^{22}$. This 
medication also affects the pathway involving IL-36y and angiogenesis. The effects of guselkumab on coronary plaque burden and cardiovascular disease in psoriasis patients has yet to be studied, but it would make a major topic for further research.

Biologic therapies currently target many of the cytokines previously mentioned, and some have been investigated for their potential cardiovascular benefits in psoriasis patients. A large Danish study of patients with severe psoriasis treated with biological agents or methotrexate showed a lower rate of cardiovascular events when compared to patients following other psoriasis treatment regimens ${ }^{23}$. When specifically considering TNF inhibitors, there is a definite reduction in the progression of plaque in men and improvement of vascular inflammation markers in both men and women ${ }^{24}$. This also correlates with a subsequent reduction in major adverse cardiovascular events ${ }^{25}$. This reduction may be linked to glycoprotein acetylation, which has recently been identified as a marker for future cardiovascular events ${ }^{26}$. Psoriasis patients with more severe disease tend to have higher levels of glycoprotein acetylation associated with subclinical cardiovascular disease, and the TNF- $\alpha$ inhibitor, adalimumab, decreases it significantly ${ }^{27,28}$. Moreover, adalimumab now has extensive safety data through the ESPRIT registry without increased incidence rates for adverse events, serious infections, or malignancies ${ }^{29}$. Therefore, clinicians can use adalimumab with confidence due to its proven safety and efficacy in treating psoriasis, with hopes that it may also have some positive effect on a patient's psoriatic cardiovascular disease.

As mentioned previously, the metabolic syndrome is more prevalent in psoriasis patients, and it is known to contribute to atherosclerosis and cardiovascular risk in general. Researchers have hypothesized that alterations in circulating adipokines such as leptin, adiponectin, and resistin were responsible for promoting metabolic syndrome in psoriasis patients ${ }^{30}$. In an effort to understand this relationship, Wolk et al. explored this topic and found that standard psoriasis treatment resulted in a decrease of resistin levels, without a significant reduction in adiponectin or leptin levels ${ }^{30}$. Further investigation is warranted in order to uncover the true clinical significance of these findings.

Despite the recent surge in research investigating cardiovascular risk in psoriasis, cumulative incidence of myocardial infarction in today's psoriatic patients remains unchanged when compared to psoriatic patients of years prior $^{31}$. As we are still unable to improve cardiovascular mortality in these patients, more research is necessary to exploit current treatment regimens for this specific indication and uncover new avenues through which medications can be developed.

\section{Summary}

In conclusion, severe psoriasis and psoriatic arthritis are associated with greater overall coronary artery plaque burden, as well as more vulnerable plaque types, when compared to controls. This explains the increased cardiovascular risk in psoriasis patients contributing to a shorter average lifespan. Many of the biologics have shown a reduction of coronary artery disease severity after prolonged treatment. However, despite this proven benefit and the extensive safety data on many of these agents, specifically adalimumab, the risk of myocardial infarction has remained the same in psoriasis patients. The need for more research to re-evaluate our current treatment recommendations for psoriasis in the context of coronary artery disease remains apparent, as does the development of specific therapies that are more targeted toward this life threatening complication of psoriasis.

\section{References}

1. Michalek IM, Loring B, John SM. A systematic review of worldwide epidemiology of psoriasis. J Eur Acad Dermatol Venereol. 2017; 31(2): 205-212. doi:10.1111/jdv.13854.

2. Takeshita J, Grewal S, Langan SM, et al. Psoriasis and Comorbid Diseases Part I. Epidemiology. Journal of the American Academy of Dermatology. 2017; 76(3): 377-390. doi:10.1016/j.jaad.2016.07.064.

3. Snekvik I, Nilsen T, Romundstad P, et al. Metabolic syndrome and risk of incident psoriasis: prospective data from the HUNT Study, Norway. British Journal of Dermatology. 2018. doi:10.1111/bjd.16885. [Epub ahead of print]

4. Mehta NN, Azfar RS, Shin DB, et al. Patients with severe psoriasis are at increased risk of cardiovascular mortality: cohort study using the General Practice Research Database. European Heart Journal. 2010; 31(8): 1000-1006. doi:10.1093/eurheartj/ehp567.

5. Mehta NN, Yu Y, Pinnelas R, et al. Attributable Risk Estimate of Severe Psoriasis on Major Cardiovascular Events. The American journal of medicine. 2011; 124(8): 775.e1-775.e6. doi:10.1016/j. amjmed.2011.03.028.

6. Fernandez-Armenteros JM, Gómez-Arbonés X, Buti-Soler M, et al. Psoriasis, Metabolic Syndrome and Cardiovascular Risk Factors. A Population-Based Study. Journal of the European Academy of Dermatology and Venereology. 2018. doi:10.1111/jdv.15159. [Epub ahead of print]

7. Lowes MA, Suarez-Farin as M, Krueger JG. Immunology of psoriasis. Annual Review of Immunol. 2014; 32: 227-255. doi:10.1146/ annurev-immunol-032713-120225.

8. Hansson GK. Inflammation, atherosclerosis, and coronary artery disease. N Engl J Med. 2005; 352: 1685-1695. doi: 10.1056/ NEJMra043430.

9. Noe MH, Shin DB, Wan MT, et al. Objective Measures of Psoriasis Severity Predict Mortality: A Prospective Population-Based Cohort Study. Journal of Investigative Dermatology. 2018; 138(1): 228 - 230. doi: 10.1016/j.jid.2017.07.841.

10. Gelfand JM, Neimann AL, Shin DB, et al. Risk of Myocardial Infarction in Patients With Psoriasis. JAMA. 2006; 296(14): 1735-1741. doi:10.1001/jama.296.14.1735.

11. Armstrong AW, Harskamp CT, Ledo L, et al. Coronary artery disease in patients with psoriasis referred for coronary angiography. Am J Cardiol. 2012; 109(7): 976-980. doi: 10.1016/j.amjcard.2011.11.025. 
12. Mansouri B, Kivelevitch D, Natarajan B, et al. Comparison of coronary artery calcium scores between patients with psoriasis and type 2 diabetes. JAMA Dermatol. 2016; 152(11): 1244-1253. doi: 10.1001/ jamadermatol.2016.2907.

13. Tawakol A, Lo J, Zanni M, et al. Increased Arterial Inflammation Relates to High-risk Coronary Plaque Morphology in HIV-Infected Patients. Journal of acquired immune deficiency syndromes (1999). 2014; 66(2): 164-171. doi:10.1097/QAI.0000000000000138.

14. Zanni MV, Abbara S, Lo J, et al. Increased Coronary Atherosclerotic Plaque Vulnerability by Coronary Computed Tomography Angiography in HIV-Infected Men. AIDS (London, England). 2013; 27(8): 1263-1272. doi:10.1097/QAD.0b013e32835eca9b.

15. Bissonnette R., Cademartiti F, Maffei E, et al. Increase in Coronary Atherosclerosis Severity and the Prevalence of Coronary Artery Mixed Plaques in Patients with Psoriasis. British Journal of Dermatology. 2017; 176(3): 800-802. doi:10.1111/bjd.14797.

16. Lerman JB, Joshi AA, Chaturvedi A, et al. Coronary plaque characterization in psoriasis reveals high-risk features that improve after treatment in a prospective observational study. Circulation. 2017; 136(3): 263-276. doi:10.1161/CIRCULATIONAHA.116.026859.

17. Shen J, Wong K, Cheng IT, et al. Increased prevalence of coronary plaque in patients with psoriatic arthritis without prior diagnosis of coronary artery disease. Annals of the Rheumatic Diseases. 2017; 76(7): 1237-1244. doi:10.1136/annrheumdis-2016-210390.

18. Chiricozzi A, Guttman-Yassky E, Suarez-Farinas M, et al. Integrative responses to IL-17 and TNF-alpha in human keratinocytes account for key inflammatory pathogenic circuits in psoriasis. J Invest Dermatol. 2011; 131(3): 677-687. doi:10.1038/jid.2010.340.

19. Bridgewood C, Fearnley GW, Keszegpal A, et al. IL-36y Is a Strong Inducer of IL-23 Production and Angiogenesis in Psoriasis. Journal of Investigative Dermatology. 2017; 137(10): S246. doi:10.1016/j. jid.2017.07.510.

20. Martinez-Lopez A, Blasco-Morente G, Perez-Lopez I, et al. Studying the Effect of Systemic and Biological Drugs on Intima-Media Thickness in Patients Suffering from Moderate and Severe Psoriasis. Journal of the European Academy of Dermatology and Venereology. 2018 Feb. doi:10.1111/jdv.14841.

21. Kim B, Lee W, Pak K, et al. Ustekinumab Treatment Is Associated with Decreased Systemic and Vascular Inflammation in Patients with Moderate to Severe Psoriasis: Feasibility Study Using 18F-Fluorodeoxyglucose Positron Emission Tomography-Computed Tomography. Journal of the American Academy of Dermatology. 2018. doi:10.1016/j.jaad.2018.03.011. [Epub ahead of print]
22. Gordon KB, Blauvelt A, Foley P, et al. Efficacy of guselkumab in subpopulations of patients with moderate-to-severe plaque psoriasis: A pooled analysis of the phase III VOYAGE 1 and VOYAGE 2 studies. British Journal of Dermatology. 2017; 178(1): 132-139.

23. Ahlehoff O, Skov L, Gislason G, et al. Cardiovascular disease event rates in patients with severe psoriasis treated with systemic antiinflammatory drugs: a Danish real-world cohort study. J Intern Med. 2013; 273(2): 197-204. doi: 10.1111/j.1365-2796.2012.02593.x.

24. Eder L, Joshi AA, Dey AK, et al. Association of Tumor Necrosis Factor Inhibitor Treatment With Reduced Indices of Subclinical Atherosclerosis in Patients With Psoriatic Disease. Arthritis \& Rheumatology. 2018; 70(3): 408-416. doi:10.1002/art.40366.

25. Wu JJ, Joshi AA, Reddy SP, et al. Anti-Inflammatory Therapy with Tumour Necrosis Factor Inhibitors Is Associated with Reduced Risk of Major Adverse Cardiovascular Events in Psoriasis. Journal of the European Academy of Dermatology and Venereology. 2018. doi:10.1111/jdv.14951. [Epub ahead of print]

26. Akinkuolie AO, Buring JE, Ridker PM, et al. A Novel Protein Glycan Biomarker and Future Cardiovascular Disease Events. Journal of the American Heart Association: Cardiovascular and Cerebrovascular Disease. 2014; 3(5): e001221. doi:10.1161/JAHA.114.001221..

27. Joshi AA, Lerman JB, Aberra TM, et al. GlycA Is a Novel Biomarker of Inflammation and Subclinical Cardiovascular Disease in Psoriasis. Circulation research. 2016; 119(11): 1242-1253. doi:10.1161/ CIRCRESAHA.116.309637.

28. Mehta NN, Shin D, Joshi A, et al. Effect of 2 Psoriasis Treatments on Vascular Inflammation and Novel Inflammatory Cardiovascular Biomarkers. Circulation: Cardiovascular Imaging. 2018; 11(6): e007394. doi:10.1161/circimaging.117.007394.

29. Menter A, Thaci D, Wu JJ, et al. Long-Term Safety and Effectiveness of Adalimumab for Moderate to Severe Psoriasis: Results from 7-Year Interim Analysis of the ESPRIT Registry. Dermatol Ther (Heidelb). 2017; 7(3): 365-381., doi:10.1007/s13555-017-0198-x.

30. Wolk K, Sabat R. Adipokines in psoriasis: an important link between skin inflammation and metabolic alterations. Rev Endocr Metab Disord. 2016; 17(3): 305-317. doi:10.1007/s11154-016-9381-0

31. Leisner MZ, Lindorff RJ, Gniadecki R, et al. Psoriasis and Risk of Myocardial Infarction before and during an Era with Biological Therapy: a Population-Based Follow-up Study. Journal of the European Academy of Dermatology and Venereology. 2018. doi:10.1111/ jdv.15021. [Epub ahead of print] 